\title{
Multilocus microsatellite analysis of European and African Candida glabrata isolates
}

ARTICLE in EUROPEAN JOURNAL OF CLINICAL MICROBIOLOGY • MARCH 2016

Impact Factor: $2.67 \cdot$ DOI: $10.1007 /$ s10096-016-2610-3

READS

76

14 AUTHORS, INCLUDING:

\section{Antonio Cascio}

Università degli Studi di Palermo

161 PUBLICATIONS 2,361 CITATIONS

SEE PROFILE

Burcu Dalyan Cilo

Uludag University

13 PUBLICATIONS 15 CITATIONS

SEE PROFILE

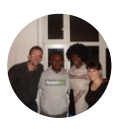

Emmanuel Nnaemeka Nnadi

Plateau State University

12 PUBLICATIONS 24 CITATIONS

SEE PROFILE

Orazio Romeo

Università degli Studi di Messina

45 PUBLICATIONS 379 CITATIONS

SEE PROFILE 


\section{Candida glabrata isolates}

\author{
V. Chillemi ${ }^{1}$ - C. L. Passo ${ }^{1}$ - A. D. van Diepeningen ${ }^{2} \cdot$ S. Rharmitt ${ }^{1}$ - D. Delfino ${ }^{3}$ \\ A. Cascio $^{3}$ - N. E. Nnadi ${ }^{4}$ B. D. Cilo ${ }^{5}$ P. Sampaio ${ }^{6}$ - H.-J. Tietz ${ }^{7}$ - J. Pemán ${ }^{8}$. \\ G. Criseo ${ }^{1} \cdot$ O. Romeo ${ }^{1,9} \cdot$ F. Scordino ${ }^{9}$
}

Abstract This study aimed to elucidate the genetic relatedness and epidemiology of 127 clinical and environmental Candida glabrata isolates from Europe and Africa using multilocus microsatellite analysis. Each isolate was first identified using phenotypic and molecular methods and subsequently, six unlinked microsatellite loci were analyzed using automated fluorescent genotyping. Genetic relationships were estimated using the minimum-spanning tree (MStree) method. Microsatellite analyses revealed the existence of 47 different genotypes. The fungal population showed an irregular distribution owing to the over-representation of genetically different infectious haplotypes. The most common genotype was MG-9, which was frequently found in both European and African isolates. In conclusion, the data reported here emphasize the role of specific $C$. glabrata genotypes in human infections for at least some decades and highlight the widespread distribution of some isolates, which seem to be more able to cause disease than others.

\section{O. Romeo}

oromeo@unime.it

1 Department of Chemical, Biological, Pharmaceutical and Environmental Sciences, University of Messina, Viale F. Stagno d'Alcontres, 31, 98166 Messina, Italy

2 CBS-KNAW Fungal Biodiversity Centre, Uppsalalaan 8, 3584 CT Utrecht, The Netherlands

3 Department of Human Pathology, University of Messina, Via Consolare Valeria, 98125 Messina, Italy

4 Department of Microbiology, Plateau State University, P.M.B 2012, Bokkos, Plateau State, Nigeria

\section{Introduction}

Candida glabrata is a haploid yeast historically believed to be harmless to humans and for a long time regarded as a nonpathogenic saprophyte of the normal flora of healthy individuals [1]. However, in recent decades, this view has been gradually weakened as a growing number of clinical reports have shown that this fungus is, on the contrary, an important pathogen [2-4].

The increase in patients with an impaired immune system, in addition to the widespread use of immunosuppressive therapies and broad-spectrum antibiotic drugs, has greatly contributed to rendering $C$. glabrata one of the most commonly isolated yeasts among all non- $C$. albicans species that infect humans [2-4]. However, although Candida albicans remains the most frequently isolated fungal pathogen in humans, C. glabrata has been reported to be an significant cause of oral infections, especially in elderly people $[4,5]$. In addition,
5 Medical Microbiology Department, Faculty of Medicine, Uludag University, 16059 Bursa, Turkey

6 Biology Department, Minho University, Campus de Gualtar, 4710-057 Braga, Portugal

7 Institut für Pilzkrankheiten, Luisenstrasse 50, 10117 Berlin, Germany

8 Servicio de Microbiología, Hospital Universitario y Politécnico La $\mathrm{Fe}$, Valencia, España

IRCCS Centro Neurolesi "Bonino-Pulejo", SS113, 98124 Messina, Italy 
this species also shows a remarkable ability to infect other human body sites including the vagina and urinary tract $[6$, 7], and in recent years it has undoubtedly emerged as the second-leading cause of bloodstream infections (BSIs) in the USA and in Northern and Central European countries [3].

The reasons for the increased isolation rate of C. glabrata are multifactorial and difficult to pinpoint exactly. However, the issue of resistance to azole derivatives $[3,8]$ and the discovery and evolution of new strains $[9,10]$ are certainly partly responsible.

For population genetics studies in C. glabrata, microsatellites have increasingly become the markers of choice [11-14]. Most microsatellites are noncoding DNA, either in intergenic sequence regions or in introns. Thus, they can generally be assumed to evolve neutrally; thus, their level of polymorphism is proportional to the underlying mutation rate.

In this study, we report the genetic profiles, obtained through analysis of six microsatellite loci, of different clinical and environmental C. glabrata isolates from Europe and Africa. Our data showed a south-north and east-west distribution of closely related genotypes and highlight the widespread distribution of particular haplotypes, which seem to be more able to cause infections than others.

\section{Materials and methods}

A total of 127 C. glabrata isolates, of different clinical and geographical origin, were examined in this study (Table 1). All isolates were initially identified by the ID 32C system (bioMeriéux, France) and subsequently confirmed using a species-specific multiplex PCR-based method that allows C. glabrata to be discriminated from its closely related species Candida bracarensis and Candida nivariensis [15, 16].

Multilocus analysis of polymorphic microsatellite markers was used to evaluate genetic relatedness among our C. glabrata isolates [11]. Six microsatellite markers were amplified by PCR using six pairs of primers, as described in Abbes et al. [11]. For automatic allele size determination the forward primer of each pair was 5'-fluorescently labelled with 6carboxyfluorescein (FAM; GLM4-Fwd, GLM6-Fwd, RPM2Fwd and MTI-Fwd primers), 6-carboxyhexafluorescein (HEX; GLM5-Fwd primer), and 6-carboxytetramethylrhodamine (TAMRA; ERG3-Fwd primer). For each isolate, six separate PCR amplifications were performed and amplicons were sent out to Eurofins-MWG/Operon (www.eurofinsdna.com) for fragment length analysis using an ABI 3130XL sequencing platform (Applied Biosystems) and GeneScan 500-ROX as the size standard. Allele binning was carried out using the program AutoBin [17]. Genetic relationships were estimated using the minimum-spanning tree (MStree) method, as implemented in BioNumerics, version 4.61 (Applied Maths, St.-MartensLatem, Belgium).
The discriminatory power $(D)$ of the microsatellite typing method used was calculated on-line (http://insilico.ehu.es/ mini_tools/discriminatory_power/index.php) using Simpson's index of diversity [18].

For statistical analysis, all isolates were split according to their geographical origin: Africa $(n=22)$, Greece $(n=15)$, Italy $(n=30)$, Portugal $(n=15)$, Spain $(n=16)$, Turkey ( $n=16)$ and Middle/Northern Europe (UK, the Netherlands, Austria, Finland, and Germany; $n=13$ ). Haplotype frequencies and genetic diversity within each population in addition to inter-population analysis were computed using the software HAPLOTYPE ANALYSIS version 1.05 (www.unigoettingen.de/en/134935.html). HAPLOTYPE ANALYSIS calculates genetic diversity within each population based on the number of different haplotypes (A) in that population, the number of unique or "private" haplotypes $(\mathrm{P})$ specific to that population, the effective number of haplotypes $(\mathrm{Ne})$ observed in the population, the estimated maximum haplotype richness or "allele richness" ( $\mathrm{Rh}$ ) defined as the number of alleles per locus and based on the rarefaction method, Nei's index of genetic diversity (He; the probability that two randomly chosen haplotypes are different) estimated without bias and the mean genetic distance between individuals $\left(\mathrm{D}_{\mathrm{sh}}{ }^{2}\right)$ as measures for genetic diversity.

Fisher's exact tests were used to test whether there were statistical differences in the proportion of the genotypes among the different countries. To better quantify the difference between the countries, we used the four clonal complexes (CCs) recognized in the 47 different microsatellite genotypes by the MStree in Fig. 1. These CCs were grouped into four classes based around the central genotypes. The classified C. glabrata populations were compared using Fisher's exact test giving us the probability that populations were similar ( $p$ values $>0.05$ ) or dissimilar $(p$ values $<0.05)$.

\section{Results}

All yeast isolates examined in this study were identified as C. glabrata isolates and no C. nivariensis or C. bracarensis were recovered. The multilocus microsatellite genotypes obtained are shown in Table 1 and Fig. 1.

A total of 84 different alleles were found for the six microsatellite loci analyzed (Table 2). Locus ERG3 showed the highest genotypic diversity with a $D$ value of 0.85 , whereas the lowest genotypic diversity was observed with the locus GLM5 ( $D=0.62$; Table 2).

When all six microsatellite markers were combined, a total of 47 diverse multilocus genotypes (MG) were obtained from 127 C. glabrata isolates (Fig. 1, Table 1). This led to an index of discrimination of 0.89 (Table 2).

One genotype (MG-9) was the most frequently observed (37 out of 127 isolates; $29 \%$ ) followed by genotypes MG-34 
Eur J Clin Microbiol Infect Dis

t1.1 Table 1 Results obtained by microsatellite genotyping analysis of the European and African C. glabrata isolates examined in this study

\begin{tabular}{|c|c|c|c|c|c|c|c|c|c|c|c|}
\hline \multirow{2}{*}{$\begin{array}{l}\mathrm{t} 1.2 \\
\mathrm{t} 1.3\end{array}$} & \multirow[t]{2}{*}{ Isolate } & \multirow[t]{2}{*}{ Origin } & \multirow[t]{2}{*}{ Year } & \multirow[t]{2}{*}{ Samples } & \multicolumn{6}{|c|}{ Microsatellite loci } & \multirow[t]{2}{*}{ Genotype } \\
\hline & & & & & ERG3 & RPM2 & MTI & GLM4 & GLM5 & GLM6 & \\
\hline $\mathrm{t} 1.4$ & CG125 & Italy & 2006 & BPS & 230 & 139 & 239 & 279 & 262 & 300 & MG-1 \\
\hline $\mathrm{t} 1.5$ & CG2018 & Italy & 2007 & Sputum & 200 & 125 & 233 & 284 & 258 & 295 & MG-17 \\
\hline $\mathrm{t} 1.6$ & CGES1 & Italy & 2012 & Sputum & 264 & 127 & 239 & 277 & 258 & 324 & MG-4 \\
\hline $\mathrm{t} 1.7$ & CGES2 & Italy & 2012 & Sputum & 207 & 133 & 239 & 265 & 258 & 324 & MG-9 \\
\hline $\mathrm{t} 1.8$ & CG2051 & Italy & 2007 & BAL & 201 & 127 & 238 & 275 & 298 & 295 & MG-20 \\
\hline $\mathrm{t} 1.9$ & CG3416 & Italy & 2008 & BAL & 207 & 133 & 239 & 265 & 258 & 324 & MG-9 \\
\hline t1.10 & CG4157 & Italy & 2009 & BAL & 207 & 133 & 239 & 265 & 258 & 324 & MG-9 \\
\hline t1.11 & $\mathrm{CG} 3285 \mathrm{~L}^{\mathrm{a}}$ & Italy & 2008 & BAL & 230 & 139 & 239 & 279 & 262 & 300 & MG-1 \\
\hline $\mathrm{t} 1.12$ & CG3285S $\mathrm{S}^{\mathrm{a}}$ & Italy & 2008 & Blood & 230 & 139 & 239 & 279 & 262 & 300 & MG-1 \\
\hline t1.13 & CG3399 & Italy & 2008 & Blood & 207 & 133 & 239 & 265 & 258 & 324 & MG-9 \\
\hline $\mathrm{t} 1.14$ & CG157 & Italy & 2006 & $\mathrm{CVC}$ & 207 & 133 & 239 & 265 & 258 & 324 & MG-9 \\
\hline $\mathrm{t} 1.15$ & CG4795 & Italy & 2009 & CVC & 264 & 127 & 239 & 280 & 258 & 324 & MG-5 \\
\hline t1.16 & CG4768 & Italy & 2009 & CVC & 264 & 127 & 239 & 280 & 258 & 324 & MG-5 \\
\hline $\mathrm{t} 1.17$ & CG1626 & Italy & 2007 & Feces & 264 & 127 & 239 & 280 & 258 & 324 & MG-5 \\
\hline t1.18 & CG1724 & Italy & 2007 & Feces & 230 & 139 & 239 & 279 & 262 & 300 & MG-1 \\
\hline t1.19 & CG1828 & Italy & 2007 & Feces & 194 & 121 & 248 & 268 & 262 & 295 & MG-25 \\
\hline $\mathrm{t} 1.20$ & CG2081 & Italy & 2007 & Feces & 264 & 127 & 239 & 277 & 258 & 324 & MG-4 \\
\hline $\mathrm{t} 1.21$ & CG2086 & Italy & 2007 & Feces & 207 & 133 & 239 & 265 & 258 & 324 & MG-9 \\
\hline $\mathrm{t} 1.22$ & CG4836 & Italy & 2009 & Feces & 264 & 127 & 239 & 277 & 258 & 324 & MG-4 \\
\hline $\mathrm{t} 1.23$ & CG1580 & Italy & 2007 & Urine & 207 & 133 & 239 & 265 & 258 & 324 & MG-9 \\
\hline $\mathrm{t} 1.24$ & CG2108 & Italy & 2007 & Urine & 237 & 121 & 239 & 265 & 258 & 287 & MG-12 \\
\hline $\mathrm{t} 1.25$ & CG2453 & Italy & 2008 & Urine & 201 & 127 & 238 & 275 & 298 & 295 & MG-20 \\
\hline $\mathrm{t} 1.26$ & CG4819 & Italy & 2009 & Urine & 207 & 133 & 239 & 265 & 258 & 324 & MG-9 \\
\hline $\mathrm{t} 1.27$ & CGLA1 & Italy & 2011 & Urine & 207 & 133 & 239 & 265 & 258 & 324 & MG-9 \\
\hline $\mathrm{t} 1.28$ & CG1721 & Italy & 2010 & Vaginal & 230 & 127 & 239 & 268 & 258 & 324 & MG-7 \\
\hline t1.29 & CG1818 & Italy & 2007 & Vaginal & 207 & 133 & 239 & 265 & 258 & 324 & MG-9 \\
\hline $\mathrm{t} 1.30$ & CG2074 & Italy & 2007 & Vaginal & 276 & 127 & 233 & 280 & 264 & 310 & MG-22 \\
\hline $\mathrm{t} 1.31$ & CG2087 & Italy & 2007 & Vaginal & 237 & 133 & 248 & 280 & 270 & 305 & MG-24 \\
\hline $\mathrm{t} 1.32$ & CG4165 & Italy & 2009 & Vaginal & 207 & 133 & 239 & 265 & 258 & 324 & MG-9 \\
\hline $\mathrm{t} 1.33$ & CG4206 & Italy & 2012 & Vaginal & 207 & 133 & 239 & 265 & 258 & 324 & MG-9 \\
\hline t1.34 & NIG10 & Nigeria & 2011 & Vaginal & 264 & 127 & 239 & 277 & 258 & 324 & MG-4 \\
\hline $\mathrm{t} 1.35$ & NIG16p & Nigeria & 2011 & Vaginal & 264 & 127 & 239 & 277 & 258 & 324 & MG-4 \\
\hline $\mathrm{t} 1.36$ & NIG19Bp & Nigeria & 2011 & Vaginal & 237 & 133 & 238 & 268 & 258 & 295 & MG-13 \\
\hline t1.37 & NIG29p & Nigeria & 2011 & Vaginal & 207 & 133 & 239 & 265 & 258 & 324 & MG-9 \\
\hline t1.38 & NIG64 & Nigeria & 2011 & Vaginal & 207 & 133 & 239 & 265 & 258 & 324 & MG-9 \\
\hline t1.39 & NIG71 & Nigeria & 2011 & Vaginal & 207 & 133 & 239 & 265 & 258 & 324 & MG-9 \\
\hline $\mathrm{t} 1.40$ & NIG108p & Nigeria & 2011 & Vaginal & 207 & 133 & 239 & 265 & 258 & 324 & MG-9 \\
\hline t1.41 & NIG154 & Nigeria & 2011 & Vaginal & 207 & 133 & 239 & 265 & 258 & 324 & MG-9 \\
\hline $\mathrm{t} 1.42$ & NIG157p & Nigeria & 2011 & Vaginal & 207 & 133 & 239 & 265 & 258 & 324 & MG-9 \\
\hline $\mathrm{t} 1.43$ & NIG162p & Nigeria & 2011 & Vaginal & 264 & 127 & 246 & 277 & 258 & 324 & MG-6 \\
\hline $\mathrm{t} 1.44$ & NIG173p & Nigeria & 2011 & Vaginal & 207 & 133 & 239 & 265 & 258 & 324 & MG-9 \\
\hline $\mathrm{t} 1.45$ & NIG176 & Nigeria & 2011 & Vaginal & 241 & 145 & 238 & 268 & 262 & 321 & MG-32 \\
\hline $\mathrm{t} 1.46$ & NIG190p & Nigeria & 2011 & Vaginal & 264 & 127 & 239 & 277 & 258 & 324 & MG-4 \\
\hline $\mathrm{t} 1.47$ & NIG194 & Nigeria & 2011 & Vaginal & 237 & 133 & 238 & 268 & 258 & 295 & MG-13 \\
\hline $\mathrm{t} 1.48$ & NIG222p & Nigeria & 2011 & Vaginal & 264 & 127 & 239 & 277 & 258 & 324 & MG-4 \\
\hline t1.49 & NIG223p & Nigeria & 2011 & Vaginal & 237 & 133 & 238 & 280 & 258 & 295 & MG-14 \\
\hline $\mathrm{t} 1.50$ & NIG244 & Nigeria & 2011 & Vaginal & 207 & 133 & 239 & 265 & 258 & 324 & MG-9 \\
\hline
\end{tabular}


t1.51 Table 1 (continued)

Isolate Origin

Year Samples

Microsatellite loci

Genotype

$\mathrm{t} 1.52$

ERG3 RPM2

MTI

GLM4

GLM5

GLM6

\begin{tabular}{|c|c|c|c|c|c|c|c|c|c|c|c|}
\hline $\mathrm{t} 1.53$ & CBS12544 & Zimbabwe & 2011 & Fruit pulp & 237 & 127 & 232 & 268 & 258 & 289 & MG-16 \\
\hline $\mathrm{t} 1.54$ & CBS2175 & UK & 1955 & Vaginal & 207 & 133 & 239 & 268 & 258 & 324 & MG-10 \\
\hline $\mathrm{t} 1.55$ & CBS2192 & South Africa & 1955 & Malt & 207 & 133 & 239 & 265 & 258 & 324 & MG-9 \\
\hline $\mathrm{t} 1.57$ & CBS4692 & Austria & - & GM & 207 & 133 & 239 & 265 & 258 & 324 & MG-9 \\
\hline $\mathrm{t} 1.58$ & CBS5040 & South Africa & - & Insect larvae & 207 & 133 & 239 & 265 & 258 & 324 & MG-9 \\
\hline $\mathrm{t} 1.59$ & CBS6144 & Finland & - & Vaginal & 230 & 127 & 239 & 268 & 258 & 324 & MG-7 \\
\hline $\mathrm{t} 1.61$ & CBS861 & Netherlands & 1942 & Mouth & 207 & 133 & 239 & 280 & 258 & 324 & MG-11 \\
\hline $\mathrm{t} 1.62$ & CBS862 & Netherlands & 1948 & Mouth & 230 & 127 & 239 & 268 & 258 & 324 & MG-7 \\
\hline $\mathrm{t} 1.63$ & CGS-65 & Spain & 2011 & Blood & 207 & 133 & 239 & 265 & 258 & 324 & MG-9 \\
\hline $\mathrm{t} 1.64$ & CGS-66 & Spain & 2011 & Blood & 207 & 133 & 239 & 265 & 258 & 324 & MG-9 \\
\hline $\mathrm{t} 1.65$ & CGS-67 & Spain & 2011 & Blood & 207 & 133 & 239 & 265 & 258 & 324 & MG-9 \\
\hline t1.69 & CGS-78 & Spain & 2013 & Blood & 230 & 127 & 239 & 268 & 258 & 324 & MG-7 \\
\hline $\mathrm{t} 1.70$ & CGS-79 & Spain & 2013 & Blood & 207 & 133 & 239 & 265 & 258 & 324 & MG-9 \\
\hline $\mathrm{t} 1.71$ & CGS-80 & Spain & 2013 & Blood & 207 & 133 & 239 & 265 & 258 & 324 & MG-9 \\
\hline $\mathrm{t} 1.72$ & CGS-81 & Spain & 2013 & Blood & 216 & 122 & 242 & 268 & 264 & 307 & MG-29 \\
\hline $\mathrm{t} 1.73$ & CGS-82 & Spain & 2013 & Blood & 194 & 121 & 247 & 268 & 260 & 295 & MG-26 \\
\hline $\mathrm{t} 1.74$ & CGS-86 & Spain & 2014 & Blood & 207 & 133 & 239 & 265 & 258 & 324 & MG-9 \\
\hline $\mathrm{t} 1.75$ & CGS-87 & Spain & 2014 & Blood & 230 & 127 & 239 & 268 & 258 & 324 & MG-7 \\
\hline $\mathrm{t} 1.76$ & CGS-88 & Spain & 2014 & Blood & 276 & 127 & 232 & 280 & 264 & 310 & MG-23 \\
\hline $\mathrm{t} 1.77$ & CGS-89 & Spain & 2014 & Blood & 215 & 127 & 225 & 268 & 282 & 321 & MG-44 \\
\hline $\mathrm{t} 1.78$ & CGS-90 & Spain & 2014 & Blood & 202 & 121 & 225 & 277 & 273 & 307 & MG-30 \\
\hline $\mathrm{t} 1.85$ & GRE1 & Greece & 2010 & Vaginal & 237 & 127 & 232 & 268 & 258 & 289 & MG-16 \\
\hline $\mathrm{t} 1.86$ & GRE2 & Greece & 2010 & Vaginal & 230 & 139 & 239 & 277 & 260 & 298 & MG-2 \\
\hline $\mathrm{t} 1.87$ & GRE3 & Greece & 2010 & Vaginal & 201 & 125 & 238 & 268 & 270 & 290 & MG-21 \\
\hline $\mathrm{t} 1.88$ & GRE5 & Greece & 2010 & Vaginal & 194 & 121 & 247 & 268 & 260 & 283 & MG-27 \\
\hline t1.89 & GRE6 & Greece & 2010 & Vaginal & 194 & 127 & 247 & 268 & 260 & 310 & MG-28 \\
\hline t1.90 & GRE8 & Greece & 2010 & Vaginal & 230 & 139 & 239 & 277 & 260 & 298 & MG-2 \\
\hline $\mathrm{t} 1.91$ & GRE9 & Greece & 2010 & Vaginal & 207 & 133 & 239 & 265 & 258 & 324 & MG-9 \\
\hline t1.92 & GRE10 & Greece & 2010 & Vaginal & 207 & 133 & 239 & 265 & 258 & 324 & MG-9 \\
\hline $\mathrm{t} 1.93$ & GRE11 & Greece & 2010 & Vaginal & 207 & 133 & 239 & 265 & 258 & 324 & MG-9 \\
\hline t1.94 & GRE12 & Greece & 2010 & Vaginal & 264 & 127 & 239 & 277 & 258 & 324 & MG-4 \\
\hline t1.95 & GRE13 & Greece & 2010 & Vaginal & 230 & 139 & 239 & 277 & 260 & 298 & MG-2 \\
\hline t1.96 & GRE16 & Greece & 2010 & Vaginal & 207 & 133 & 239 & 265 & 258 & 324 & MG-9 \\
\hline $\mathrm{t} 1.97$ & GRE17 & Greece & 2010 & Vaginal & 230 & 127 & 239 & 268 & 258 & 324 & MG-7 \\
\hline t1.98 & GRE18 & Greece & 2010 & Vaginal & 264 & 127 & 239 & 277 & 258 & 324 & MG-4 \\
\hline t1.99 & GRE19 & Greece & 2010 & Vaginal & 215 & 127 & 225 & 265 & 279 & 321 & MG-45 \\
\hline
\end{tabular}


Eur J Clin Microbiol Infect Dis

t1.100 Table 1 (continued)

Isolate Origin $\quad$ Year $\quad$ Samples $\quad$ Microsatellite loci

Genotype

\begin{tabular}{|c|c|c|c|c|c|c|c|c|c|c|}
\hline \multirow{2}{*}{$\mathrm{t} 1.101$} & & & & & \\
\hline & & & & ERG3 & RPM2 & MTI & GLM4 & GLM5 & GLM6 & \\
\hline t1.102 SAN1B & Morocco & 2015 & Vaginal & 216 & 134 & 238 & 271 & 260 & 298 & MG-31 \\
\hline t1.103 SAN2 & Morocco & 2015 & Vaginal & 235 & 125 & 230 & 263 & 256 & 287 & MG-37 \\
\hline t1.104 HSJ118 & Portugal & 2005 & Blood & 205 & 131 & 238 & 280 & 256 & 321 & MG-33 \\
\hline t1.105 8B & Portugal & 2004 & Urine & 205 & 131 & 238 & 263 & 256 & 321 & MG-34 \\
\hline t1.106 CIPO55 & Portugal & 2005 & Feces & 205 & 131 & 238 & 263 & 256 & 321 & MG-34 \\
\hline t1.108 HSJ42 & Portugal & 2005 & Blood & 205 & 131 & 238 & 263 & 256 & 321 & MG-34 \\
\hline t1.109 CIPO88 & Portugal & 2005 & Vaginal & 205 & 131 & 238 & 263 & 262 & 318 & MG-35 \\
\hline t1.110 HSJ177 & Portugal & 2005 & Blood & 205 & 131 & 238 & 263 & 256 & 321 & MG-34 \\
\hline t1.111 HSJ117 & Portugal & 2005 & Blood & 205 & 131 & 238 & 263 & 256 & 321 & MG-34 \\
\hline t1.112 CIPO103 & Portugal & 2005 & $\mathrm{RF}$ & 228 & 125 & 237 & 265 & 256 & 321 & MG-38 \\
\hline t1.117 HSJ55 & Portugal & 2005 & Blood & 205 & 131 & 238 & 263 & 256 & 321 & MG-34 \\
\hline t1.118 CIPO44 & Portugal & 2005 & Feces & 205 & 131 & 238 & 263 & 256 & 321 & MG-34 \\
\hline t1.119 TUR1 & Turkey & 2014 & Blood & 198 & 125 & 236 & 272 & 295 & 292 & MG-46 \\
\hline t1.120 TUR2 & Turkey & 2013 & Blood & 205 & 131 & 238 & 263 & 256 & 321 & MG-34 \\
\hline t1.121 TUR3 & Turkey & 2014 & Urine & 228 & 137 & 237 & 275 & 258 & 295 & MG-18 \\
\hline t1.122 TUR4 & Turkey & 2014 & Urine & 228 & 125 & 231 & 265 & 270 & 295 & MG-43 \\
\hline t1.123 TUR5 & Turkey & 2013 & Urine & 228 & 125 & 237 & 265 & 256 & 321 & MG-38 \\
\hline t1.124 TUR6 & Turkey & 2014 & Urine & 262 & 130 & 231 & 275 & 258 & 295 & MG-19 \\
\hline t1.125 TUR7 & Turkey & 2013 & Urine & 205 & 131 & 238 & 263 & 256 & 321 & MG-34 \\
\hline t1.133 TUR16 & Turkey & 2014 & Urine & 243 & 125 & 237 & 268 & 256 & 321 & MG-40 \\
\hline t1.134 TUR20 & Turkey & 2014 & Blood & 228 & 125 & 237 & 265 & 256 & 321 & MG-38 \\
\hline
\end{tabular}

$B P S$ biopsy of the paravertebral space, $B A L$ bronchoalveolar lavage, $C V C$ central venous catheter, $G M$ case of generalized mycosis, $G I$ gastrointestinal, $R F$ respiratory fluid, $C S F$ cerebrospinal fluid

${ }^{\mathrm{a}}$ Same patient

148 (13 out of $127 ; 10 \%)$, MG-4 (11 out of $127 ; \sim 9 \%$ ) and MG-7

149 (7 out of $127 ; 5 \%$ ). The remaining 43 genotypes were found

150 with an incidence of below $5 \%$ (Table 1). In total, we ana-

151 lyzed 51 vaginal samples, 30 blood samples, and 17 from

152 urine as the three most common sample types, whereas the

153 remaining 29 were from a variety of other specimens.

154 However, statistical analysis showed no significant associa-

155 tion between the microsatellite genotypes and the origin or

156 date of isolation of our isolates.
The predominant genotype MG-9 was found in 35 clinical samples from different countries and in two environmental samples (insect larvae and malt) in South Africa. Most of the MG-9 isolates were found in Italian (12 out of 37; $32 \%$ ) and African (10 out of $37 ; 27 \%$ ) specimens, whereas the remaining isolates were spread over Spain, Greece, and the Middle/ Northern European countries (Fig. 1). Interestingly, another closely related genotype (MG-4), was also frequently encountered in Italy ( 3 out of $11 ; 27 \%$ ) and Africa ( 4 out of $11 ; 36 \%$; 


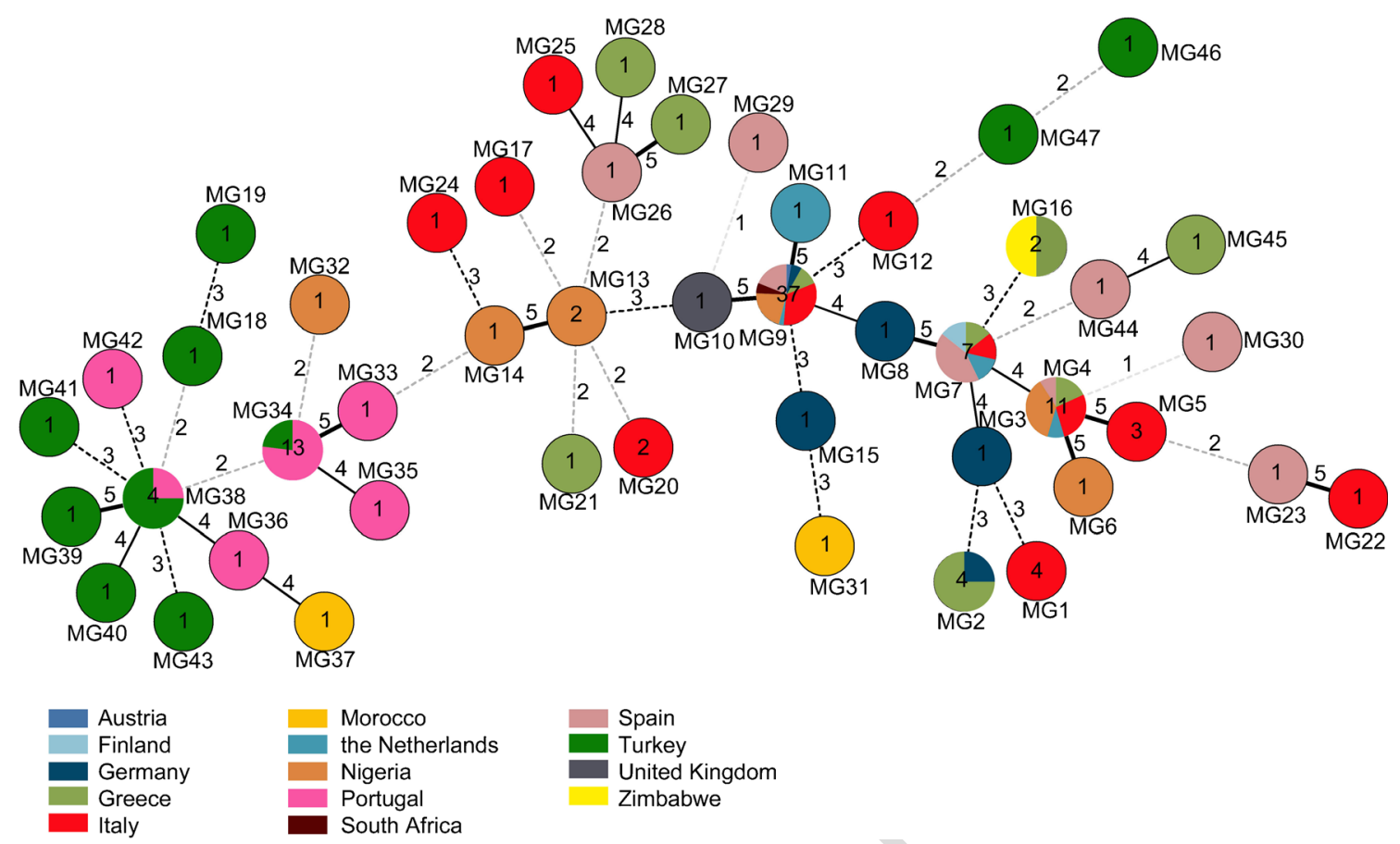

Fig. 1 Minimum spanning tree showing the differences based on a categorical analysis between the genotypes. Each circle represents a unique haplotype with the number of isolates of that genotype inside subdivided by country of origin based on the colors. Numbers at the

166

167

168

169

170

171
Fig. 1, Table 1). Conversely, the second most common genotype (MG-34) found in this study was exclusively recovered from Portuguese and Turkish clinical samples. This clone, together with the MG-38 genotype and a number of closely related genetic variants, form a particular cluster of isolates evolutionarily distinct from other European isolates (Fig. 1).

All our observed microsatellite genotypes form a large network in the MStree analysis with no outliers (Fig. 1). However, several related CCs or microsatellite classes can be defined based on the central, dominant genotypes. For our analysis, we defined four clonal clusters $(\mathrm{CC} 1, \mathrm{CC} 2$, $\mathrm{CC} 3$, and $\mathrm{CC} 4$ ) with the following central types MG-34, MG-13, MG-9, and MG-7 respectively (Fig. 1). The CC1, with MG-34 as the central type, contains the genotypes MG18, MG-19, and MG-32 to MG-43; CC2, with MG-13 as the central type, contains MGs 14, 17, 20, 21, and 24-28; CC3, with MG-9 as the central type, includes MGs from 8 to 12,15 , connecting lines correspond to the number of identical microsatellites between the isolates. Similarities between genotypes were visualized using BioNumerics version 4.61 treating the data as categorical information

29, 31, 46, and 47, while the remainder (MGs 1-7, 16, 22, 23, 30,44 , and 45 ) fall into CC4, with MG-7 as the central type (Fig. 1).

According to their geographical origin, our isolates can be subdivided into seven different populations (Table 3). Even though the populations differed in their sample sizes, the number of haplotypes detected in each population and the numbers of private haplotypes specific to a given population did not differ very much. Portugal and Spain were the two countries with the lowest haplotypic richness and diversity, whereas the highest were in Middle/Northern Europe and Turkey (Table 3). However, a normalized pairwise population matrix of Nei's genetic distance showed that Portugal and Turkey in particular were more distant from the other countries.

The populations of Africa, Middle/Northern Europe, Italy, Greece, and Spain did not differ significantly from one another ( $p$ values $>0.05)$ whereas the Portuguese and Turkish
183
Table 2 Number of alleles and index of diversity for each microsatellite marker examined in this study

\begin{tabular}{llllllll}
\hline & \multicolumn{2}{l}{ Microsatellite loci } & & & Total \\
\cline { 2 - 6 } & ERG3 & RPM2 & MTI & GLM4 & GLM5 & GLM6 & \\
\hline Number of alleles & 19 & 12 & 14 & 11 & 13 & 15 & 84 \\
Range size (bp) & $181-260$ & $121-139$ & $227-247$ & $261-288$ & $259-307$ & $280-325$ & - \\
Diversity index & 0.85 & 0.78 & 0.64 & 0.79 & 0.62 & 0.70 & 0.89 \\
\hline
\end{tabular}


Eur J Clin Microbiol Infect Dis

Table 3 Population analysis

t3.2 based on the microsatellite

Q2

$\mathrm{t} 3.3$

t3.4

$\mathrm{t} 3.5$

$\mathrm{t} 3.6$

$\mathrm{t} 3.7$

t3.8

t3.9

t3.10 haplotypes

\begin{tabular}{llllllll}
\hline Population & $n$ & $\mathrm{~A}$ & $\mathrm{P}$ & $\mathrm{Ne}$ & $\mathrm{Rh}$ & $\mathrm{He}$ & $\mathrm{D}_{\text {sh }}{ }^{2}$ \\
\hline Africa & 22 & 9 & 6 & 3,841 & 5,372 & 0775 & 627,636 \\
Greece & 15 & 9 & 4 & 6,429 & 7,190 & 0905 & 822,749 \\
Italy & 30 & 11 & 8 & 4,787 & 5,865 & 0818 & 884,636 \\
Middle/Northern & 13 & 9 & 5 & 6,259 & 8,000 & 0910 & 361,863 \\
$\quad$ Europe & 15 & 6 & 4 & 2,143 & 4,333 & 0571 & 643,863 \\
Portugal & 16 & 8 & 5 & 4,000 & 5,873 & 0800 & 657,771 \\
Spain & 16 & 10 & 8 & 7,529 & 7,682 & 0925 & 860,621 \\
Turkey & $18 \pm 5.9$ & $9 \pm 1.6$ & $5.7 \pm 1.7$ & $5.0 \pm 1.9$ & $6.3 \pm 1.3$ & $0.81 \pm 0.12$ & $694 \pm 182$ \\
Mean \pm SD & & & & & & &
\end{tabular}

$N$ sample size in each population, $A$ number of haplotypes detected in each population, $P$ number of private haplotypes, $\mathrm{Ne}$ effective number of haplotypes, $R h$ haplotypic richness, $\mathrm{He}$ genetic diversity, $D_{s h}{ }^{2}$ mean genetic distance between individuals, $S D$ standard deviation populations differed significantly from all others ( $p$ values of $6.7 \mathrm{e}-4$ and smaller), but not between themselves ( $p$ value $=0.330$ ).

\section{Discussions and conclusions}

In recent years, important changes in the epidemiology of candidiasis have been observed worldwide, highlighting a significant increase in non-C. albicans species with different degrees of virulence and pathogenicity $[3,15]$. Among these species, C. glabrata has emerged as an important cause of human disease, proving to possess an extraordinary ability to infect different body sites [3-7, 19]. However, based on our results, it seems that not all C. glabrata isolates are equally as likely to cause human infections and some of them, especially those with the MG-9 haplotype, appear to be clinically most relevant and cause disease significantly more often than others (Fig. 1). This remarkable capacity of some isolates to infect different host tissues may be the result of their unique genetic background (Table 1). This observation agrees with previous studies $[12,14]$ and suggests that the identification of specific genotypes could represent an important step for future studies on the virulence of $C$. glabrata.

In this study, the combined analysis of the six microsatellite markers reached a $D$ value of 0.89 and the 127 C. glabrata isolates were divided into 47 different genotypes. Our $D$ value was not consistent with that reported by Abbes et al. [11], and it is below the desirable value of 0.95 for an "ideal" typing system [20]. Therefore, our results suggest that the present markers should be used with caution if utilized in an attempt to recognize outbreaks of infections, but they can be used for some population studies.

In this study, we were able to recognize at least four CCs. Looking at the distribution of these clonal complexes over the different geographical regions sampled, we observed an expansion of closely related genotypes (MG-9, MG-7, and MG-
4) that were prevalent in southern countries (Italy and Spain) and in Africa and were probably spread by the huge flow of migrants that occurs between these two continents. Conversely, two closely related genotypes, MG-34 and MG38, were restricted only to Turkey and Portugal, even if they showed distantly genetic relationships with the African types MG-37, MG-32, and MG-14. This latter, together with the other Nigerian MG-13, correlates more directly with the main type, MG-9.

The MG-9 type was the most frequently observed genotype spreading between Africa and European countries for long periods of time. In fact, infections caused by this genotype date back to more than 80 years ago (1935) as demonstrated by some $C$. glabrata strains in the CBS culture collection that were isolated during the two decades between 1935 and 1955 (Table 1).

All other clinical isolates were recovered during or after 2004 and the MG-9 genotype was still present in recent clinical samples by demonstrating its exceptional, strong adaptation to specific geographical areas. Hence, the MG-9 seems to be the main European type, while its branching out to other main genotypes via the German MG-8 and the UK sample MG-10 suggests that these branches might have been crucial to the expansion of $C$. glabrata in Europe.

Even though in part, the same set of microsatellite loci was used in other $C$. glabrata population studies, it proves difficult to directly compare the results, as small size differences can be observed in the dominant types. These could be due to actual size differences or to errors in the measurements. To avoid such problems in the future we advocate the use of a standard set of reference strains, available from public sources. For this purpose we deposited a representative panel of strains (MGs $7,9,13$, and 34 in this study) to the CBS culture collection (www.cbs.knaw.nl) in Utrecht, the Netherlands, and the strains are also available from the corresponding author.

In conclusion, the data reported here emphasize the role of specific $C$. glabrata genotypes in human infections for at least 
a few decades. We recommend further genetic studies that may detect a different reality than that currently known with regard to the population structure, epidemiology, and spread of this fungal pathogen.

\section{Compliance with ethical standards}

Funding This research was supported in part by the EU Mare Nostrum (EUMN-III Call) program of the European Union, grant agreement number 2011-4050/001-EMA2. Dr Sanae Rharmitt was the recipient of a scholarship (10 months) signed within the EUMN program for PhD students (F.S. 1.04.11.01 UORI) under the supervision of Prof Orazio Romeo.

Conflicts of interest The authors declare that they have no conflicts of interest.

Ethical approval This article does not contain any studies with human participants or animals performed by any of the authors.

Informed consent For this type of study formal consent is not required.

\section{References}

1. Bolotin-Fukuhara M, Fairhead C (2014) Candida glabrata: a deadly companion? Yeast 31:279-288. doi:10.1002/yea.3019

2. Pfaller MA, Andes DR, Diekema DJ, Horn DL, Reboli AC, Rotstein C, Franks B, Azie NE (2014) Epidemiology and outcomes of invasive candidiasis due to non-albicans species of Candida in 2, 496 patients: data from the Prospective Antifungal Therapy (PATH) registry 2004-2008. PLoS One 9, e101510. doi:10.1371/journal. pone.0101510

3. Quindós G (2014) Epidemiology of candidaemia and invasive candidiasis. A changing face. Rev Iberoam Micol 31:42-48. doi:10. 1016/j.riam.2013.10.001

4. Silva S, Negri M, Henriques M, Oliveira R, Williams DW, Azeredo J (2012) Candida glabrata, Candida parapsilosis and Candida tropicalis: biology, epidemiology, pathogenicity and antifungal resistance. FEMS Microbiol Rev 36:288-305. doi:10.1111/j.15746976.2011.00278.x

5. Li L, Redding S, Dongari-Bagtzoglou A (2007) Candida glabrata: an emerging oral opportunistic pathogen. J Dent Res 86:204-215. doi:10.1177/154405910708600304

6. Tomczak H, Szałek E, Grześkowiak E (2014) The problems of urinary tract infections with Candida spp. aetiology in women. Postepy Hig Med Dosw (Online) 68:1036-1039. doi:10.5604/ 17322693.1118989

7. Sardi JC, Scorzoni L, Bernardi T, Fusco-Almeida AM, Mendes Giannini MJ (2013) Candida species: current epidemiology, pathogenicity, biofilm formation, natural antifungal products and new therapeutic options. J Med Microbiol 62:10-24. doi:10.1099/jmm. $0.045054-0$
8. Pfaller MA, Castanheira M, Messer SA, Moet GJ, Jones RN (2010) Variation in Candida spp. distribution and antifungal resistance rates among bloodstream infection isolates by patient age: report from the SENTRY Antimicrobial Surveillance Program (20082009). Diagn Microbiol Infect Dis 68:278-283. doi:10.1016/j. diagmicrobio.2010.06.015

9. Turner SA, Butler G (2014) The Candida pathogenic species complex. Cold Spring Harb Perspect Med 4:a019778. doi:10.1101/ cshperspect.a019778

10. Chapeland-Leclerc F, Hennequin C, Papon N, Noël T, Girard A Socié G, Ribaud P, Lacroix C (2010) Acquisition of flucytosine, azole, and caspofungin resistance in Candida glabrata bloodstream isolates serially obtained from a hematopoietic stem cell transplant recipient. Antimicrob Agents Chemother 54:1360-1362. doi:10. 1128/AAC.01138-09

11. Abbes S, Sellami H, Sellami A, Hadrich I, Amouri I, Mahfoudh N, Neji S, Makni F, Makni H, Ayadi A (2012) Candida glabrata strain relatedness by new microsatellite markers. Eur J Clin Microbiol Infect Dis 31:83-91. doi:10.1007/s10096-011-1280-4

12. Amouri I, Sellami H, Abbes S, Hadrich I, Mahfoudh N, Makni H, Ayadi A (2012) Microsatellite analysis of Candida isolates from recurrent vulvovaginal candidiasis. J Med Microbiol 61:10911096. doi:10.1099/jmm.0.043992-0

13. Brisse S, Pannier C, Angoulvant A, de Meeus T, Diancourt L, Faure O, Muller H, Peman J, Viviani MA, Grillot R, Dujon B, Fairhead C, Hennequin C (2009) Uneven distribution of mating types among genotypes of Candida glabrata isolates from clinical samples. Eukaryot Cell 8:287-295. doi:10.1128/EC.00215-08

14. Foulet F, Nicolas N, Eloy O, Botterel F, Gantier JC, Costa JM, Bretagne $S$ (2005) Microsatellite marker analysis as a typing system for Candida glabrata. J Clin Microbiol 43:4574-4579. doi:10. 1128/JCM.43.9.4574-4579.2005

15. Criseo G, Scordino F, Romeo O (2015) Current methods for identifying clinically important cryptic Candida species. J Microbiol Methods 111:50-56. doi:10.1016/j.mimet.2015.02.004

16. Romeo O, Scordino F, Pernice I, Lo Passo C, Criseo G (2009) A multiplex PCR protocol for rapid identification of Candida glabrata and its phylogenetically related species Candida nivariensis and Candida bracarensis. J Microbiol Methods 79: 117-120. doi:10.1016/j.mimet.2009.07.016

17. Guichoux E, Lagache L, Wagner $\mathrm{S}$, Chaumeil P, Léger P, Lepais $\mathrm{O}$, Lepoittevin C, Malausa T, Revardel E, Salin F, Petit RJ (2011) Current trends in microsatellite genotyping. Mol Ecol Resour 11: 591-611. doi:10.1111/j.1755-0998.2011.03014.x

18. Hunter PR, Gaston MA (1998) Numerical index of the discriminatory ability of typing systems: an application of Simpson's index of diversity. J Clin Microbiol 26:2465-2466

19. Esposto MC, Prigitano A, Romeo O, Criseo G, Trovato L, Tullio V, Fadda ME, Tortorano AM; FIMUA Working Group (2013) Looking for Candida nivariensis and C. bracarensis among a large Italian collection of $C$. glabrata isolates: results of the FIMUA working group. Mycoses 56:394-396. doi:10.1111/myc. 12026

20. Van Belkum A, Tassios PT, Dijkshoorn L, Haeggman S, Cookson B, Fry NK, Fussing V, Green J, Feil E, Gerner-Smidt P, Brisse S, Struelens M (2007) Guidelines for the validation and application of typing methods for use in bacterial epidemiology. Clin Microbiol Infect 13:1-46. doi:10.1111/j.1469-0691.2007.01786.x
317 